\title{
Difficulty Balancing and Review Management System for Smart Mobile Learning
}

\author{
Youngjae Chun, Kiho Youm, Kyoungsu Oh \\ Department of Media, Soongsil University \\ \{dkreformer, youmkh010,oks\}@ssu.ac.kr
}

\begin{abstract}
In this paper, we introduce an efficient learning management system for mobile learning. The system consists of dynamic difficulty adjustment and review management. Difficulty balancing and review at appropriate time enhance learning performance. Learner's learning ability and difficulty of learning are quantified by rating system used in computer game. The ratings are adjusted dynamically every learning for difficulty balancing. Review periods based on forgetting curve is useful to memorize something. Proposed system makes learners sustain memory for a long time by providing personalized review management. In addition, learners can share their own learning progress with friends through social network services. This activity can increase motivation on learning. The proposed system can be exploited for any types of learnings by customizing learning style.
\end{abstract}

Keywords: Mobile learning, Serious game, Gamification, Dynamic difficulty adjustment, Forgetting curve, Social network services

\section{Introduction}

The performance of recent mobile devices such as smart phones and the wireless internet environment has improved, so much research is currently investigating mobile learning. Some mobile learning applications are following effective study methods which are suggested by Dunlosky et al [1]. However, the methods do not consider the ability of learners, and use the same study environment. Consequently, the learning efficiency may be different for each user.

In this paper, we introduce dynamic difficulty adjustment and a review management system for education using mobile devices. Since our system is for mobile learning, it provides the effective learning study methods mentioned in [1]. Simultaneously, the system adjusts the selection criteria adaptively for a question to solve depending on the user's ability. The dynamic adjustment of the learning difficulty is similar to difficulty adjustment systems in computer games, and it helps maintain users' interest in learning. In addition, personalized learning environment is provided to each user according to individual difficulty criteria. Our learning system also makes users review through a verified review period. Learners do not need to bother with scheduling when they should do a review themselves. Learners can use our smart mobile learning system by simply studying with the mobile learning application whenever they want.

In addition, our system gives learners feedback similar to computer games when they answer questions correctly or their learning skill reaches a certain level. It is a good example of the gamification of mobile learning with difficulty balancing. The proposed system is applied for a prototype of an English vocabulary memorization study, and it can be exploited for more general learning expanding exam-styles. Moreover, learners can share their own learning progress with their neighbors through social network services (SNS) to increase their motivation.

This paper contains the following sections: Section 2 discusses previous research studies and approaches related to difficulty adjustment in computer games and mobile learning. Section 3 will introduce an overview of the proposed system. Dynamic difficulty adjustment and review management are explained in section 4 and 5, respectively. Section 6 shows the 
SNSs connected to our system. Finally, our contribution, the proposed methods, and its applicable areas are summarized.

\section{Related Work}

The objective of the game is to immerse the user in the game for a lot of time. Elements in games to achieve this objective can be utilized in the positive aspect. Recently, through their experiments, many studies verified that the computer game for the learning is very effective([2]-[4]). It is extremely important that the immersion characteristics of the game can be applied to improve performance of learning. Interaction in games and rewards given after successful game playing are the basic elements of gamification. In particular, the application of difficulty balancing can enhance the degree and flow of a learner's learning.

Dynamic Difficulty Adjustment [5] has explained that it is possible to make a player feel entertained and spend a lot of time playing a game by adjusting the difficulty dynamically according to the player's skill. If a game is too easy for a player than they will be bored, and too much difficulty will frustrate them; therefore, learners should study something at an appropriate difficulty level to sustain their interest in learning.

Personalized context-aware ubiquitous learning systems that support effective English vocabulary learning [6] suggested a method that considers a learner's location, cultural situation, and lifestyle for the efficient learning of English words [6]. The learner's ability to use English words is assessed using Item Response Theory (IRT) [7]. However, the method focused on context-aware recommendations rather than difficulty of learning.

A personalized mobile English vocabulary learning system [8] introduced a mobile learning system that recommends proper English words tailored to the learner's ability. Bayesian estimation is exploited to estimate the learner's skill level, and the difficulty of words is determined by their frequency, length, and pronunciation. The drawback of this approach is that the difficulty of the words cannot be changed once it computed. In particular, the frequency of words can vary according to the new contents such as articles, SNS posts etc. In addition, there is no association with SNS, even though they researched mobile learning systems.

SNS has many benefits as a motivational tool in mobile learning. Many research studies have addressed the fact that students' learning efficiency can be enhanced via Twitter to form a community similar to a classroom $([9 ; 10 ; 11]$ Dunlap \& Lowenthal, 2009; Evans, 2013; Junco, Elavsky, \& Heiberger, 2012). In this paper, our system uses the Facebook SNS to share learning progress with neighbors. The ability to express themselves with their learning performance makes learners spend more time and improves their efforts to improve their performance. Additional motivation can be occur through competition in good faith among neighbors who are using the same mobile learning application.

\section{System Overview}

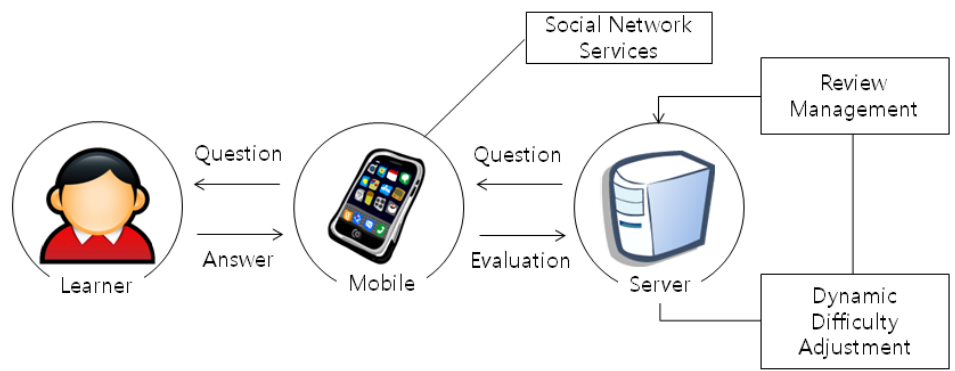

Figure 1. System Overview 
In the proposed system, a mobile device receives a question from a server, and a learner solves the exam-style question on the mobile device (Figure 1). Exam-style question solving is an effective method for learning as [1] mentions. Since we used a mobile device, learners can study whenever and wherever they wish. These properties also represent effective learning methods. Figure 2 shows the prototype executed on a mobile device. A learner solves the given question by selecting the letters that correspond to the blanks. In our system, a learner solves the questions which are sent from our server. However, the learner's skill and the difficulty of the questions can be dynamically adjusted while the learner just solves the questions. Because of the adjustment, a learner will receive questions that are suitable for their learning ability. If there is a specific question that a learner has to review, our system selects it and send it to the learner.
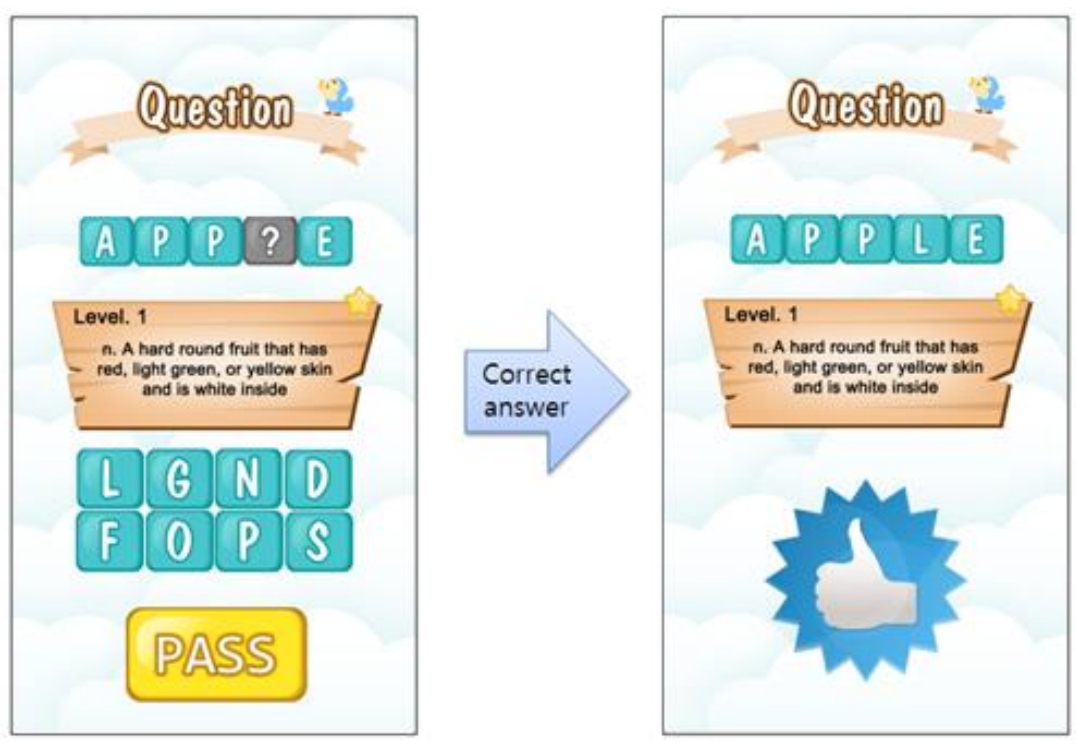

Figure 2. Prototype Scenes

The proposed system is based on providing suitable questions to a learner and making sure a learner reviews at the appropriate time. When a learner solves a question, depending on the evaluation of the answer, the system adjusts the learner's learning skill and the difficulty of the questions. After dynamic difficulty adjustment, a new question that is appropriate to the learner's level is given. In order to remember solutions for a long time, a review after a reasonable time is necessary. Our system helps learners efficiently memorize questions by providing them with review periods. The review periods are based on the forgetting curve is exploited to keep information in not the short-term memory but the long-term memory.

The mobile learning application's workflow is shown in Figure 3. First, a learner logs into the system using their user ID and password. The user information for the system is used to specify the difficulty of the questions. The system automatically gives the learner a new question or a review question. After answering, the mobile application evaluates the answer and transmits the evaluated result to the server. The proposed system adjusts to the learner's ability and the difficulty of the questions based on the evaluated results. 


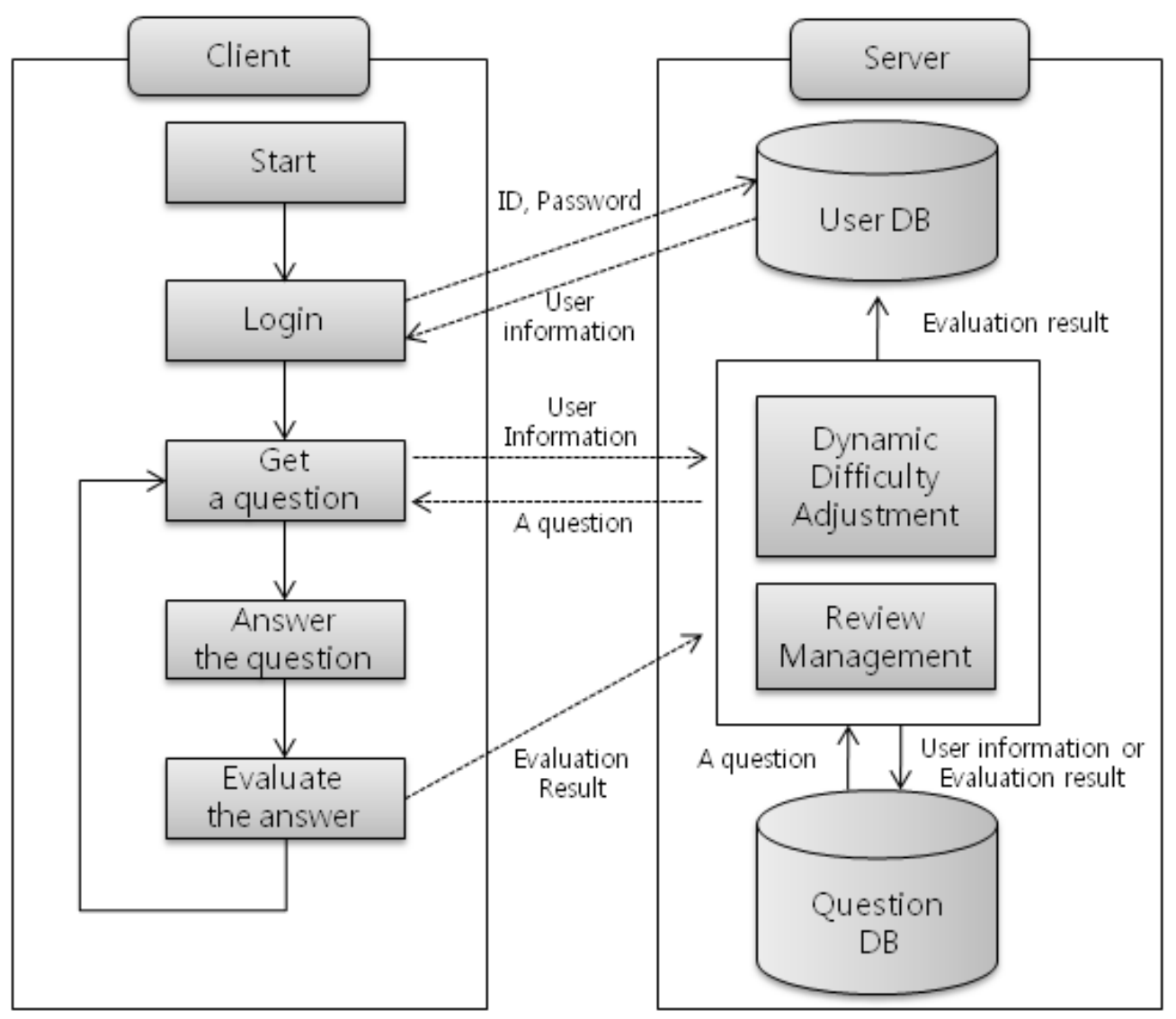

Figure 3. Workflow of our Mobile Learning Application

\section{Dynamic Difficulty Adjustment in Mobile Learning}

The difficulty of the game is one of the most important elements for determining how immersive a game can become. It is very difficult to determine a difficulty that satisfies a wide spectrum of users. Thus, many studies into dynamic difficulty adjustment have been performed. In this paper, we consider a given question that a learner needs to solve as a challenge and our work focuses on finding the proper challenge. Interest in the study can be maintained if our system provides a question that is suitable to a learner's level.

The glicko-2 rating system [12] is a method used in games such as chess to evaluate the strength of user's skill in the game. Rating indicates the ability of users, and ratings deviation (RD) means the accuracy of the rating. The rating is a population mean, and the RD is a population standard deviation from the normal distribution. For example, the level of a user who has a 1000 rating and $100 \mathrm{RD}$ is between 800 and 1200 , with a probability of $95.5 \%$ (figure 4). After a user has played a game, that user's rating and RD can be updated according to their opponent's rating and RD. The RD will increase if the user does not play a game for a while. 


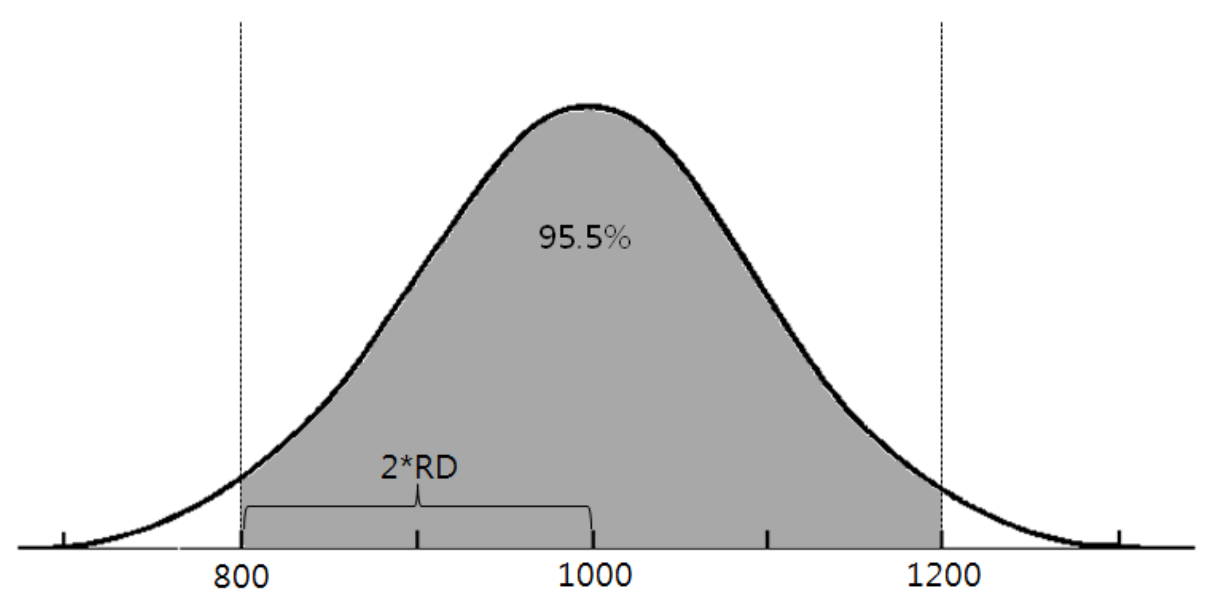

Figure 4. Normal Distribution of Learning Ability Presented by Rating and RD

The proposed system uses the rating and RD of the glicko- 2 system to quantify the learning capabilities of learners. The difficulty of each question is also quantified by the rating system and is shared by all learners. In the same way as in a chess game, a learner's rating increases and difficulty rating of a question increases when the learner solves the question correctly. Conversely, a learner's rating decreases and the difficulty rating of a question increases when the learner solves a question incorrectly. The rating and difficulty are only changed when a learner solves a question for the first time. In other words, rating and difficulty are not changed during a review. If the correct answer rate of a question is high, it can be assumed that the inherent difficulty rating of that question is low.

Choosing a question that has an appropriate difficulty can be performed by selecting a question that has a similar difficulty to the learner's rating. Because the ability of a learner is within the scope such as in figure 4, when our system needs to select a new question, it selects a question that corresponds to the range. In the proposed system, we do not use the standard deviation for questions, and the RD of questions is assumed to zero. Furthermore, if a learner has not used the system for a long time, their RD increases.

\section{Review Management based on Forgetting Curve}

To remember what has been learned for longer, reviews are necessary. Ebbinghaus [13], asserted that the probability that you remember something with precision decreases over time after its first memorization. Tony Buzan [14] studied the review period based on Ebbinghaus's theory. According to [14], reviews should be executed in periods such as in figure 5. Through such a system, it is possible to maintain memory for a long time.

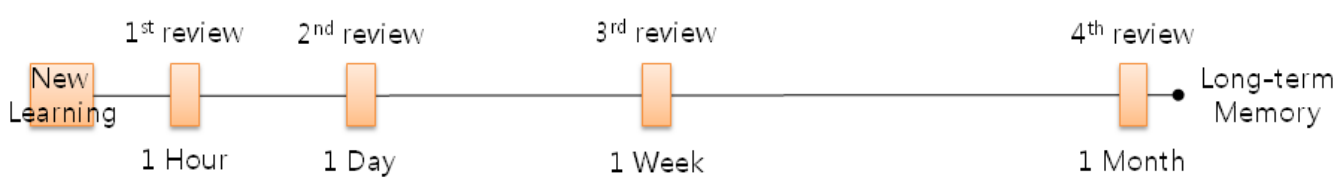

Figure 5. Review Periods

Our system leads learners to review using the repetition cycles of figure 5. If a question the user needs to review is found, it is given to the learner instead of giving them a new one. Each question for each learner has different review period. Therefore, the proposed system saves 
which questions are solved, reviewed or never solved for each learner. For each question, the time taken to answer successfully, and how long each learner has to wait for the next review are saved.

$\mathrm{T}_{\mathrm{e}}$ in equation (1) is the difference between the last learning time and the current time. When $T_{e}$ is greater than the review period $T_{r}$, a review is required. During learning, the number of new questions decreases and the amount of reviews increases. Thus, it is necessary to find the question most urgently needed to review from all questions for review. For this, our system computes importance of a review $\mathrm{R}$ using equation (2). The importance of review increases as $T_{e}$ increases; however, the effect of $T_{e}$ on the importance of review is reduced as $\mathrm{T}_{\mathrm{r}}$ increases. In other words, the questions that have the same $\mathrm{T}_{\mathrm{e}}$ also depend on the number of reviews, and the importance of the review will differ with respect to this. Our review management system can select a question that significantly requires review by considering the importance $\mathrm{R}$. The update of the review period of a question is performed when a learner answers a question correctly. If a learner submits an incorrect answer to a question, the question will be repeated after the learner solves a further 10 questions.

$$
\begin{gathered}
\mathrm{T}_{\mathrm{e}}=\mathrm{T}_{\mathrm{c}}-\mathrm{T}_{\mathrm{s}} \quad\left(\mathrm{T}_{\mathrm{c}}>\mathrm{T}_{\mathrm{s}}\right) \\
\mathrm{R}=\left(\mathrm{T}_{\mathrm{e}}-\mathrm{T}_{\mathrm{r}}\right) / \mathrm{T}_{\mathrm{r}} \quad\left(\mathrm{T}_{\mathrm{e}} \geq \mathrm{T}_{\mathrm{r}}\right)
\end{gathered}
$$

\section{Social Network Service}

Motivation plays a major role in the learning process. Sharing the results of learning with friends can make a learner proud of their learning abilities. This can serve as a motivation to induce continued learning. As mentioned in the related research, social network services (SNSs) are the ideal space for sharing results. Furthermore, while group of learners who use the learning application are connected via a social network, good faith competition can be generated and the motivation to learn is further increased. Learners can use Facebook, a largescale SNS, to post their weekly learning results. In figure 6 , the scores that have been acquired through learning are shown, as are the number of questions that the learner has studied per week. Positive competition and boasting, which are used in games to improve participants' enjoyment, can make users more eager to continue playing, and we can apply this concept to immerse our learners in learning. This method is considered a part of the gamification of learning.
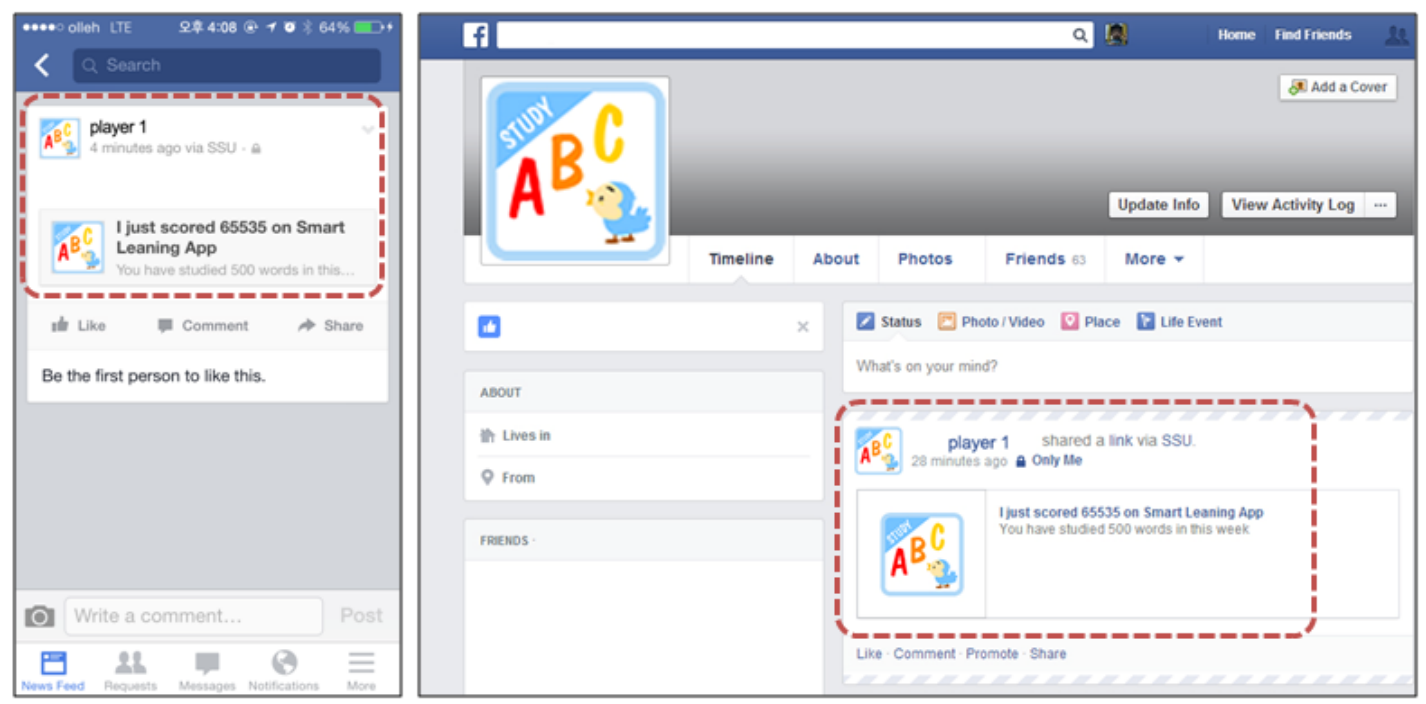

Figure 6. Sharing Learning Progress and Performance via SNS 


\section{Conclusion}

In this paper, we have introduced a mobile learning system based on review management and dynamic adjustment of question difficulty. The system maintains user's interest in learning by providing questions suitable for their ability. In order to estimate the difficulty of questions and the ability of learners, our system uses a rating system to evaluate the power games such as chess. The manner in which the selection of the questions corresponds to the ability of the user is similar to balancing systems in computer games. This is a good example of applying the characteristics of games to learning. Since our review management based on the forgetting curve is internally operated, learners do not worry about reviews. Furthermore, by using a mobile device, learners can learn in any place at any desired time. Additionally, by sharing their own learning performance through SNSs, it is possible to motivate themselves further. Our system is applied to the prototype for learning English words, but it can be used for all kinds of learning by expanding the exam style.

\section{Acknowledgements}

This work was supported by the Korea Science and Engineering Foundation(KOSEF) grant funded by the Korea government(MEST) (No.2011-0012214).

\section{References}

[1] J. Dunlosky, K. A. Rawson, E. J. Marsh, M. J. Nathan and D. T. Willingham, “Improving students' learning with effective learning techniques promising directions from cognitive and educational psychology", Psychological Science in the Public Interest, vol. 14, no. 1, (2013), pp. 4-58.

[2] F. Ke andB. Grabowski, "Gameplaying for maths learning: cooperative or not?", British Journal of Educational Technology, vol. 38, (2007), pp. 249-259.

[3] M. Virvou, G, Katsionis and K, Manos, "Combining software games with education: Evaluation of its educational effectiveness", Educational Technology \& Society, vol. 8, no. 2, (2005), pp. 54-65.

[4] W. L. Wong, C. Shen, L. Nocera, E. Carriazo, F. Tang, S. Bugga and H. Narayanan, "Serious video game effectiveness", Proceedings of the international conference on Advances in computer entertainment technology, vol. 36, no. 4, (2007), pp. 49-55.

[5] O. Missura, "Dynamic Difficulty Adjustment", (2014).

[6] C. M. Chen and Y. L. Li, "Personalised context-aware ubiquitous learning system for supporting effective English vocabulary learning”, Interactive Learning Environments, vol. 18, no. 4, (2010), pp. 341-364.

[7] F. B. Baker, "Item response theory: Parameter estimation techniques", New York: Marcel Dekker, (1992).

[8] C. M. Chen and C. J. Chung, "Personalized mobile English vocabulary learning system based on item response theory and learning memory cycle", Computers \& Education, vol. 51, no. 2, (2008), pp. 624-645.

[9] J. C. Dunlap and P. R. Lowenthal, "Horton hears a tweet", Educause Quarterly, vol. 32, no. 4, (2009), pp. 111.

[10] C. Evans, "Twitter for teaching: Can social media be used to enhance the process of learning?", British Journal of Educational Technology, (2013), pp. 1-14.

[11] R. Junco, C. M. Elavsky and G. Heiberger, "Putting Twitter to the test: Assessment outcomes for student collaboration, engagement and success", British Journal of Educational Technology, vol. 44, no. 2, (2012), pp. 272-287.

[12] M. E. Glickman, "Parameter estimation in large dynamic paired comparison experiments", Journal of the Royal Statistical Society: Series C (Applied Statistics), vol. 48, no. 3, (1999), pp. 377-394.

[13] H. Ebbinghaus, "Über das gedächtnis: untersuchungen zur experimentellen psychologie", Duncker \& Humblot, (1885).

[14] T. Buzan, "Use your head", Rajpal \& Sons, (1982). 


\section{Authors}

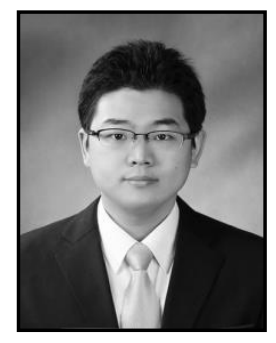

Youngjae Chun. He received the B.S degree in Departmant of Media from Soongsil University in 2007 and he is working on the Ph.D. degree in Department of Media from Soongsil University. His research interests include Real-time rendering, Computer game, serious game and Image Processing.

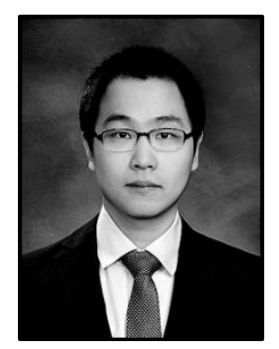

Kiho Youm. He received the B.S degree in Departmant of Media from Soongsil University in 2011 and he is working on the M.S. degree in Department of Media from Soongsil University. His research interests include Real-time rendering, Computer game, serious game and Image Processing.

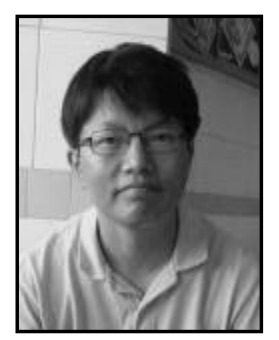

Kyoungsu Oh. He received B.E., M.E., and Ph.D. degrees from Seoul National University in 1994, 1996, and 2001 respectively. After that, he worked at ZOI MENT from 2001 to 2002 . He is currently an associate professor of Soongsil University. His main research areas are computer graphics, computer game, media art and serious game. 